\title{
THE BLOW-UP SOLUTIONS TO NONLINEAR FRACTIONAL DIFFERENTIAL CAPUTO-SYSTEM
}

\author{
M. Terchi ${ }^{1}$, H. Hassouna ${ }^{1}$ \\ ${ }^{1}$ Biskra University, Biskra, Algeria \\ E-mails: terchi1.messaouda@gmail.com, houda.hassouna@yahoo.fr
}

\begin{abstract}
In this paper, we establish the finite time blow-up of solutions to nonlinear differential systems governed by Caputo fractional differential equation. Then, we derive sufficient conditions on parameters with positive given data. Moreover, for this purpose under some assumptions, we prove the non existence of global solutions to the considered class of nonlinear fractional differential Caputo-system subject to the initial condition. To prove our main result, we apply the test function method, Riemann-Liouville integral, Caputo derivative operator and some general analysis tools. Our result is new and generalizes the existing one.

Keywords: blow-up; differential equations; Riemann-Liouville integral; Caputo derivative operator.
\end{abstract}

\section{Introduction}

The original conception of fractional calculus was appeared prior to the turn of 20th century, when L'Hopital wrote a letter to Leibniz asking him about a particular notation he had used in his application for the $n$ th-derivative of the function $f(x)=x$. L'Hopital posed the question to Leibniz about the result when $n=\frac{1}{2}$. From that time, fractional calculus become the most famous notation attracted more and more mathematicians and physicists who have discovered many disciplines, because fractional differential equations provide an excellent tool used to describe various phenomena such as rheology, damping phenomena, diffusion processes, etc. From this fact, we have many definitions of the concept of noninteger order integral or derivative according to the notations and methodology of the researchers, and the most common are given by Riemann-Liouville and Caputo which will define later. The difference between these two definitions is connected with the order of calculation.

The study of fractional ordinary differential systems have been widely used in different fields of science, and the existence and uniqueness of local solutions to the integral equations are proved by many researchers, see [1-11] and the references therein. Recently, Abbas [1] establishes the existence of solutions to fractional order ordinary and delay differential equations with some applications. In [2], Mahto, Abbas and Favini use Sadovskii's fixed point method to investigate the existence and uniqueness of solutions to Caputo impulsive fractional differential equations. Mahto and Abbas [3] introduce a PCalmost automorphic function and establish the composition theorem. As an application, the authors study the existence of almost automorphic solution to impulsive fractional functional differential equations under the condition that the forcing term is almost automorphic.

Our main objective is to find finite time blow-up of solutions to the following Caputo fractional differential equation: 


$$
\left\{\begin{array}{l}
u_{t}(t)+{ }^{c} D_{0^{+}}^{\alpha_{1}} u(t)+{ }^{c} D_{0^{+}}^{\beta_{1}} u(t)=D_{0^{+}}^{\gamma_{1}}|v(t)|^{q}, \\
v_{t}(t)+{ }^{c} D_{0^{+}}^{\alpha_{2}} v(t)+{ }^{c} D_{0^{+}}^{\beta_{2}} v(t)=D_{0^{+}}^{\gamma_{2}}|u(t)|^{p}, \quad t \in \mathbb{R}_{+}, \\
u(0)=u_{0}, v(0)=v_{0} .
\end{array}\right.
$$

where $p, q>1, u_{0}, v_{0}>0,0<\alpha_{i}, \beta_{i}, \gamma_{i}<1, \alpha_{i}<\beta_{i}$ and , $i=1,2$. Our result is new and compliment the existing one.

In order to motivate our results, it is convenient to recall some facts about the fractional ordinary differential system. In [12], the authors study the profile of blowing-up solution to nonlinear system of fractional differential equations (FDE in short):

$$
\left\{\begin{array}{l}
u^{\prime}(t)+{ }^{c} D_{0^{+}}^{\alpha}(u)(t)=|v(t)|^{q} \\
v^{\prime}(t)+{ }^{c} D_{0^{+}}^{\beta}(v)(t)=|u(t)|^{p}, \quad t \in \mathbb{R}_{+}, \\
u(0)=u_{0}>0, v(0)=v_{0}>0
\end{array}\right.
$$

and prove that the solutions to the precedent problem blow-up in finite time, if $1-\frac{1}{p q}<$ $\alpha+\frac{\beta}{p}$ or $1-\frac{1}{p q}<\beta+\frac{\alpha}{p}$.

In [13], the authors multiply the caputo derivatives terms ${ }^{c} D_{0^{+}}^{\alpha}(u)(t)$ and ${ }^{c} D_{0^{+}}^{\beta}(v)(t)$ by the continuous functions $A(t), B(t)$, respectively, and obtain the following system:

$$
\left\{\begin{array}{c}
u^{\prime}(t)+A(t)^{c} D_{0^{+}}^{\alpha}(u)(t)=|v(t)|^{q}, \\
v^{\prime}(t)+B(t)^{c} D_{0^{+}}^{\beta}(v),(t)=|u(t)|^{p}, \quad t \in \mathbb{R}_{+}, \\
u(0)=u_{0}>0, v(0)=v_{0}>0 .
\end{array}\right.
$$

Under the condition $h=\min \left\{T,\left(\frac{\Gamma(2-\alpha)}{2 M}\right)^{\frac{1}{1-\alpha}},\left(\frac{\Gamma(2-\beta)}{2 M}\right)^{\frac{1}{1-\beta}}, \frac{\left(\frac{c}{2}\right)^{1 / q}-v_{0}}{c}, \frac{\left(\frac{c}{2}\right)^{1 / p}-u_{0}}{c}\right\}$, the authors of the work [13] guarantee the existence of local solution to their problem. Then, the authors used Lipschitz condition on $A(t)$ and $B(t)$ to prove the blowing-up results.

After that, the authors of the paper [8] generalize the system of Qun and Huilai [13]. The authors take $A(t)=\sum_{i=1}^{n} a_{i}(t), B(t)=\sum_{i=1}^{n} b_{i}(t)$, and express the right hand side of the system by the Riemann-Liouville operator of the functions $f$ and $g$. The problem takes the following form:

$$
\left\{\begin{array}{l}
u_{t}(t)+a_{1}(t)^{c} D_{0^{+}}^{\alpha}(u)(t)+\ldots+\alpha_{n}^{c} D_{0^{+}}^{\alpha}(u)(t)=J_{0^{+}}^{1-\gamma_{1}} f(u, v), \\
v_{t}(t)+b_{1}(t)^{c} D_{0^{+}}^{\beta}(v)(t)+\ldots+b_{n}(t)^{c} D_{0^{+}}^{\beta}(v)(t)=J_{0^{+}}^{1-\gamma_{2}} g(u, v), \quad t \in \mathbb{R}_{+} \\
u(0)=u_{0}>0, v(0)=v_{0}>0,
\end{array}\right.
$$

where $p, q>1,0<\alpha_{i}<1,0<b_{i}<1, i=1, . ., n, f$ and $g$ are arbitrary analytical functions. Their method of proof is based on Arzela Ascoli and Shauder theorems for the existence of local solution. Then, the authors use the weak formulation of the systems with a good choice of test function for the blowing up result, for more details see [8].

In this paper, we mix the two precedents systems. We take $n=2, J_{0^{+}}^{1-\gamma_{1}} f(u, v)=$ $D_{0^{+}}^{\gamma_{1}}|v(t)|^{q}, J_{0^{+}}^{1-\gamma_{2}} g(u, v)=D_{0^{+}}^{\gamma_{2}}|u(t)|^{p}, a_{1}(t)=a_{2}(t)=b_{1}(t)=b_{2}(t)=1$ to obtain system (1). We determine the sufficient conditions on parameters of system (1) to show the non existence of global solution to system (1). The paper is organised as follows. In Section 2 , we present definitions, properties of fractional derivative equations, and some lemmas needed for our work. Section 3 is devoted to proof of the main results on the blow up in finite time of solutions to system (1). 


\section{Some Preliminary Results}

In this section, we present several lemmas for the proof of Theorem 1 and Theorem 2. Let $A C[0, T]$ be the space of all functions which are absolutely continuous on $[0, T]$, where $0<T<\infty$. Then, for $f \in A C[0, T]$, the left-handed and right-handed Riemann-Liouville fractional derivatives $D_{0^{+}}^{\alpha} f(t)$ and $D_{T-}^{\alpha} f(t)$ of order $\alpha \in(0,1)$ are defined as follows:

$$
\begin{aligned}
& D_{0^{+}}^{\alpha} f(t)=\frac{1}{\Gamma(1-\alpha)} \frac{d}{d t} \int_{0}^{t}(t-s)^{-\alpha} f(s) d s, t \in[0, T], \\
& D_{T-}^{\alpha} f(t)=\frac{-1}{\Gamma(1-\alpha)} \frac{d}{d t} \int_{t}^{T}(s-t)^{-\alpha} f(s) d s, t \in[0, T] .
\end{aligned}
$$

It is shown in [5, Lemma 2.2], see also [6], that, for $f \in A C([0, T])$,

$$
D_{0^{+}}^{\alpha} f(t)=\frac{1}{\Gamma(1-\alpha)}\left[t^{-\alpha} f(0)+\int_{0}^{t}(t-s)^{-\alpha} f^{\prime}(s) d s\right] .
$$

Note that

$$
D_{0^{+}}^{\alpha}(f(t)-f(0))(t)=\frac{1}{\Gamma(1-\alpha)} \int_{0}^{t}(t-s)^{-\alpha} f^{\prime}(s) d s:={ }^{c} D_{0^{+}}^{\alpha} f(t),
$$

which is the Caputo derivative of $f$. For the right-hand derivative, we have

$$
D_{T-}^{\alpha} f(t)=\frac{1}{\Gamma(1-\alpha)}\left[\frac{f(T)}{(T-t)^{\alpha}}-\int_{t}^{T}(t-s)^{-\alpha} f(s) d s\right] .
$$

In $[5$, Corollary 2, p. 46], it is shown that, for the functions $f, g \in C([0, t])$ the derivatives $D_{0^{+}}^{\alpha} g, D_{T-}^{\alpha} f$ exist at every point of $[0, T]$ and are continuous. We have

$$
\int_{0}^{T} f(t) D_{0^{+}}^{\alpha} g(t) d t=\int_{0}^{T} D_{T-}^{\alpha} f(t) g(t) d t
$$

which is the formula for integration by parts.

Lemma 1. Let $\varphi(t)$ be a function such that

$$
\varphi(t)= \begin{cases}(1-t / T)^{\lambda}, & 0 \leq t \leq T, \quad \lambda \geq 2 . \\ 0, & t>T,\end{cases}
$$

Then we have

$$
\int_{0}^{T} D_{T-}^{\alpha} \varphi(t) d t=K_{\alpha, \lambda} T^{1-\alpha}, \quad K_{\alpha, \lambda}=\frac{\lambda \Gamma(\lambda-\alpha)}{(\lambda-\alpha+1) \Gamma(\lambda-2 \alpha+1)} .
$$

Lemma 2. Let $\varphi(t)$ be a function such that

$$
\varphi(t)= \begin{cases}(1-t / T)^{\lambda}, & 0 \leq t \leq T, \quad \lambda \geq 2, \\ 0, & t>T,\end{cases}
$$


and suppose that $p>1$. Then, for $\lambda>p-1$,

$$
\int_{0}^{T} \varphi^{1-p}(t)\left|\varphi^{\prime}(t)\right|^{p} d t=\Lambda_{p} T^{1-p}, \quad \Lambda_{p}=\frac{\lambda^{p}}{\lambda-p+1}
$$

and, for $\lambda>\alpha p-1$

$$
\int_{0}^{T} \varphi^{1-p}(t)\left|D_{T-}^{\alpha} \varphi(t)\right|^{p} d t=\Lambda_{p, \alpha} T^{1-\alpha p}, \quad \Lambda_{p, \alpha}=\frac{\lambda^{p}}{\lambda-\alpha p+1}\left[\frac{\Gamma(\lambda-\alpha)}{\Gamma(\lambda-2 \alpha+1)}\right]^{p} .
$$

\section{Blow-up Results}

This section is devoted to proof of the blow-up of solution to system (1).

Theorem 1. Let $T>0$, assume that

$$
\begin{gathered}
\gamma_{2}>\max \left(\alpha_{2}, \beta_{2}\right) \text { or } \alpha_{2}<\gamma_{2}<\beta_{2}, \\
\gamma_{2}+\gamma_{1}<\min \left\{1+\alpha_{2}, 1+\beta_{2}, \beta_{1}+\frac{\beta_{2}}{2}+\frac{1}{p q}-1\right\}, \\
1<q<\min \left\{\frac{1-\gamma_{1}}{\gamma_{2}-\alpha_{2}}, \frac{1-\gamma_{1}}{\gamma_{2}-\beta_{2}}\right\} .
\end{gathered}
$$

Then all solutions $(u, v) \in C([0, T])$ to (1) blow-up in finite time.

Proof. Let us carry out the proof by contradiction. Suppose that $(u ; v)$ is a non trivial weak solution to (1), which exists globally in time. First, we have

$$
{ }^{c} D u=D_{0^{+}}\left(u-u_{0}\right) .
$$

Then, we can rewrite problem (1) as follows:

$$
\left\{\begin{array}{l}
u_{t}+D_{0^{+}}^{\alpha_{1}}\left(u-u_{0}\right)+D_{0^{+}}^{\beta_{1}}\left(u-u_{0}\right)=D_{0^{+}}^{\gamma_{1}}|v|^{q}, \\
v_{t}+D_{0^{+}}^{\alpha_{2}}\left(v-v_{0}\right)+D_{0^{+}}^{\beta_{2}}\left(v-v_{0}\right)=D_{0^{+}}^{\gamma_{2}}|u|^{p}, \\
u(0)=u_{0}, v(0)=v_{0} .
\end{array}\right.
$$

Let $T>0$, then

$$
\int_{0}^{T} u_{t} \varphi d t+\int_{0}^{T} D_{0^{+}}^{\alpha_{1}}\left(u-u_{0}\right) \varphi d t+\int_{0}^{T} D_{0^{+}}^{\beta_{1}}\left(u-u_{0}\right) \varphi d t=\int_{0}^{T} D_{0^{+}}^{\gamma_{1}}|v|^{q} \varphi d t
$$

and

$$
\int_{0}^{T} v_{t} \varphi d t+\int_{0}^{T} D_{0^{+}}^{\alpha_{2}}\left(v-v_{0}\right) \varphi d t+\int_{0}^{T} D_{0^{+}}^{\beta_{2}}\left(v-v_{0}\right) \varphi d t=\int_{0}^{T} D_{0^{+}}^{\gamma_{2}}|u|^{p} \varphi d t .
$$

By using (4) and simple calculations we can obtain

$$
\begin{aligned}
& \int_{0}^{T}|v|^{q} D_{T^{-}}^{\gamma_{1}} \varphi d t+u_{0}\left(1+\int_{0}^{T} D_{T^{-}}^{\alpha_{1}} \varphi d t+\int_{0}^{T} D_{T^{-}}^{\beta_{1}} \varphi d t\right)= \\
& =\int_{0}^{T} u D_{T^{-}}^{\alpha_{1}} \varphi d t+\int_{0}^{T} u D_{T^{-}}^{\beta_{1}} \varphi d t-\int_{0}^{T} u \varphi^{\prime} d t,
\end{aligned}
$$


and

$$
\begin{aligned}
& \int_{0}^{T}|u|^{p} D_{T^{-}}^{\gamma_{2}} \varphi d t+v_{0}\left(1+\int_{0}^{T} D_{T^{-}}^{\alpha_{2}} \varphi d t+\int_{0}^{T} D_{T^{-}}^{\beta_{2}} \varphi d t\right)= \\
& =\int_{0}^{T} v D_{T^{-}}^{\alpha_{2}} \varphi d t+\int_{0}^{T} v D_{T^{-}}^{\beta_{2}} \varphi d t-\int_{0}^{T} v \varphi^{\prime} d t .
\end{aligned}
$$

Applying Hölder's inequality with parameters $p$ and $p^{\prime}$ (rsp. $q$ and $q^{\prime}$ ) to the right hand sides of (8) and (9), we derive

$$
\begin{aligned}
\int_{0}^{T} u D_{T^{-}}^{\alpha_{1}} \varphi d t & =\int_{0}^{T} u\left(D_{T^{-}}^{\gamma_{2}} \varphi\right)^{1 / p}\left(D_{T^{-}}^{\gamma_{2}} \varphi\right)^{-1 / p} D_{T^{-}}^{\alpha_{1}} \varphi d t \leq \\
& \leq\left(\int_{0}^{T}|u|^{p}\left(D_{T^{-}}^{\gamma_{2}} \varphi\right) d t\right)^{1 / p}\left(\int_{0}^{T}\left(D_{T^{-}}^{\gamma_{2}} \varphi\right)^{-p^{\prime} / p}\left|D_{T^{-}}^{\alpha_{1}} \varphi\right|^{p^{\prime}} d t\right)^{1 / p^{\prime}} \\
\int_{0}^{T} u D_{T^{-}}^{\beta_{1}} \varphi d t & =\int_{0}^{\gamma_{0}} u\left(D_{T^{-}}^{\gamma_{2}} \varphi\right)^{1 / p}\left(D_{T^{-}}^{\gamma_{2}} \varphi\right)^{-1 / p} D_{T^{-}}^{\beta_{1}} \varphi d t \leq \\
& \left.\leq \int_{0}^{T}|u|^{p}\left(D_{T^{-}}^{\gamma_{2}} \varphi\right) d t\right)^{1 / p}\left(\int_{0}^{T}\left(D_{T^{-}}^{\gamma_{2}} \varphi\right)^{-p / p^{\prime}}\left|D_{T^{-}}^{\beta_{1}} \varphi\right|^{p^{\prime}} d t\right)^{1 / p^{\prime}} \\
\int_{0}^{T} u \varphi^{\prime} d t & \leq\left(\int_{0}^{\gamma_{T}}|u|^{p}\left(D_{T^{-}}^{\gamma_{2}} \varphi\right) d t\right)^{1 / p}\left(D_{T^{-}}^{\gamma_{2}} \varphi\right)^{-1 / p} \varphi^{\prime} d t \leq \\
& \left.\int_{0}^{T}\left(D_{T^{-}}^{\gamma_{2}} \varphi\right)^{-p^{\prime} / p}\left|\varphi^{\prime}\right|^{p^{\prime}} d t\right)^{1 / p^{\prime}},
\end{aligned}
$$

and

$$
\begin{aligned}
\int_{0}^{T} v D_{T^{-}}^{\alpha_{2}} \varphi d t & =\int_{0}^{T} v\left(D_{T^{-}}^{\gamma_{1}} \varphi\right)^{1 / q}\left(D_{T^{-}}^{\gamma_{1}} \varphi\right)^{-1 / q} D_{T^{-}}^{\alpha_{2}} \varphi d t \leq \\
& \leq\left(\int_{0}^{T}|v|^{q}\left(D_{T^{-}}^{\gamma_{1}} \varphi\right) d t\right)^{1 / q}\left(\int_{0}^{T}\left(D_{T^{-}}^{\gamma_{1}} \varphi\right)^{-q^{\prime} / q}\left|D_{T^{-}}^{\alpha_{2}} \varphi\right|^{q^{\prime}} d t\right)^{1 / q^{\prime}}, \\
\int_{0}^{T} v D_{T^{-}}^{\beta_{2}} \varphi d t & =\int_{0}^{T} v\left(D_{T^{-}}^{\gamma_{1}} \varphi\right)^{1 / q}\left(D_{T^{-}}^{\gamma_{1}} \varphi\right)^{-1 / q} D_{T^{-}}^{\beta_{2}} \varphi d t \leq \\
& \left.\leq \int_{0}^{T}|v|^{q}\left(D_{T^{-}}^{\gamma_{1}} \varphi\right) d t\right)^{1 / q}\left(\int_{0}^{T}\left(D_{T^{-}}^{\gamma_{1}} \varphi\right)^{-q^{\prime} / q}\left|D_{T^{-}}^{\beta_{2}} \varphi\right|^{q^{\prime}} d t\right)^{1 / q^{\prime}}, \\
\int_{0}^{T} v \varphi^{\prime} d t & \int_{0}^{T} v\left(D_{T^{-}}^{\gamma_{1}} \varphi\right)^{1 / q}\left(D_{T^{-}}^{\gamma_{1}} \varphi\right)^{-1 / q} \varphi^{\prime} d t \leq \\
& \leq\left(\int_{0}^{T}|v|^{q}\left(D_{T^{-}}^{\gamma_{1}} \varphi\right) d t\right)^{1 / q}\left(\int_{0}^{T}\left(D_{T^{-}}^{\gamma_{1}} \varphi\right)^{-q^{\prime} / q}\left|\varphi^{\prime}\right|^{q^{\prime}} d t\right)^{1 / q^{\prime}} .
\end{aligned}
$$


Let

$$
\begin{aligned}
I_{1} & =\int_{0}^{T}|u|^{p}\left(D_{T^{-}}^{\gamma_{2}} \varphi\right) d t, & J_{1} & =\int_{0}^{T}|v|^{q}\left(D_{T^{-}}^{\gamma_{1}} \varphi\right) d t \\
I_{2} & =\int_{0}^{T}\left(D_{T^{-}}^{\gamma_{2}} \varphi\right)^{-p^{\prime} / p}\left|D_{T^{-}}^{\alpha_{1}} \varphi\right|^{p^{\prime}} d t, & J_{2} & =\int_{0}^{T}\left(D_{T^{-}}^{\gamma_{1}} \varphi\right)^{-q^{\prime} / q}\left|D_{T^{-}}^{\alpha_{2}} \varphi\right|^{q^{\prime}} d t \\
I_{3} & =\int_{0}^{T}\left(D_{T^{-}}^{\gamma_{2}} \varphi\right)^{-p^{\prime} / p}\left|D_{T^{-}}^{\beta_{1}} \varphi\right|^{p^{\prime}} d t, & J_{3} & =\int_{0}^{T}\left(D_{T^{-}}^{\gamma_{1}} \varphi\right)^{-q^{\prime} / q}\left|D_{T^{-}}^{\beta_{2}} \varphi\right|^{q^{\prime}} d t \\
I_{4} & =\int_{0}^{T}\left(D_{T^{-}}^{\gamma_{2}} \varphi\right)^{-p^{\prime} / p}\left|\varphi^{\prime}\right|^{p^{\prime}} d t, & J_{4} & =\int_{0}^{T}\left(D_{T^{-}}^{\gamma_{1}} \varphi\right)^{-q^{\prime} / q}\left|\varphi^{\prime}\right|^{q^{\prime}} d t .
\end{aligned}
$$

Then, we can rewrite (8) and (9) as

$$
J_{1}+u_{0}\left(1+\int_{0}^{T} D_{T^{-}}^{\alpha_{1}} \varphi d t+\int_{0}^{T} D_{T^{-}}^{\beta_{1}} \varphi d t\right) \leq I_{1}^{1 / p}\left(I_{2}^{1 / p^{\prime}}+I_{3}^{1 / p^{\prime}}+I_{4}^{1 / p^{\prime}}\right)
$$

and

$$
I_{1}+v_{0}\left(1+\int_{0}^{T} D_{T^{-}}^{\alpha_{2}} \varphi d t+\int_{0}^{T} D_{T^{-}}^{\beta_{2}} \varphi d t\right) \leq J_{1}^{1 / q}\left(J_{2}^{1 / q^{\prime}}+J_{3}^{1 / q^{\prime}}+J_{4}^{1 / q^{\prime}}\right) .
$$

Using (10) and (11), we obtain the inequalities

$$
J_{1} \leq I_{1}^{1 / p}\left(I_{2}^{1 / p^{\prime}}+I_{3}^{1 / p^{\prime}}+I_{4}^{1 / p^{\prime}}\right)
$$

and

from which we have

$$
I_{1} \leq J_{1}^{1 / q}\left(J_{2}^{1 / q^{\prime}}+J_{3}^{1 / q^{\prime}}+J_{4}^{1 / q^{\prime}}\right)
$$

$$
J_{1}^{1-1 / p q} \leq\left(I_{2}^{1 / p^{\prime}}+I_{3}^{1 / p^{\prime}}+I_{4}^{1 / p^{\prime}}\right)\left(J_{2}^{1 / q^{\prime}}+J_{3}^{1 / q^{\prime}}+J_{4}^{1 / q^{\prime}}\right)^{1 / p} .
$$

Next, using Lemmas 1 and 2 in the right-hand side of (14), we find

$$
\begin{aligned}
J_{1}^{1-1 / p q} & \leq C\left(T^{\left(\frac{\gamma_{2}}{p}-1\right)+\frac{1}{p^{\prime}}}+T^{\left(\frac{\gamma_{2}}{p}-\alpha_{1}\right)+\frac{1}{p^{\prime}}}+T^{\left(\frac{\gamma_{2}}{p}-\beta_{1}\right)+\frac{1}{p^{\prime}}}\right) \\
& \leq\left(T^{\left(\frac{\gamma_{1}}{q}-1\right)+\frac{1}{q^{\prime}}}+T^{\left(\frac{\gamma_{1}}{q}-\alpha_{2}\right)+\frac{1}{q^{\prime}}}+T^{\left(\frac{\gamma_{1}}{q}-\beta_{2}\right)+\frac{1}{q^{\prime}}}\right)^{1 / p} .
\end{aligned}
$$

Then, we can write

$$
J_{1}^{1-1 / p q} \leq C\left(T^{S_{1}}+T^{S_{2}}+T^{S_{3}}\right)\left(T^{S_{4}}+T^{S_{5}}+T^{S_{6}}\right),
$$

where

$$
\begin{aligned}
& S_{1}=\left(\frac{\gamma_{2}}{p}-1\right)+\frac{1}{p^{\prime}} ; \quad S_{2}=\left(\frac{\gamma_{2}}{p}-\alpha_{1}\right)+\frac{1}{p^{\prime}} ; \quad S_{3}=\left(\frac{\gamma_{2}}{p}-\beta_{1}\right)+\frac{1}{p^{\prime}} ; \\
& S_{4}=\left[\left(\frac{\gamma_{1}}{q}-1\right)+\frac{1}{q^{\prime}}\right] \frac{1}{p} ; \quad S_{5}=\left[\left(\frac{\gamma_{1}}{q}-\alpha_{2}\right)+\frac{1}{q^{\prime}}\right] \frac{1}{p} ; \quad S_{6}=\left[\left(\frac{\gamma_{1}}{q}-\beta_{2}\right)+\frac{1}{q^{\prime}}\right] \frac{1}{p} .
\end{aligned}
$$


Therefore,

$$
J_{1}^{1-1 / p q} \leq C\left(\begin{array}{c}
T^{S_{1}+S_{4}}+T^{S_{1}+S_{5}}+T^{S_{1}+S_{6}}+T^{S_{2}+S_{4}}+T^{S_{2}+S_{5}}+ \\
+T^{S_{2}+S_{6}}+T^{S_{3}+S_{4}}+T^{S_{3}+S_{5}}+T^{S_{3}+S_{6}}
\end{array}\right) .
$$

Note that $S_{1}+S_{4}<0, S_{1}+S_{4}<S_{1}+S_{5}, S_{1}+S_{4}<S_{1}+S_{6}$, while $S_{2}+S_{4}<S_{2}+S_{5}$ $S_{2}+S_{4}<S_{2}+S_{6}$, and $S_{3}+S_{4}<S_{3}+S_{5}, S_{3}+S_{4}<S_{3}+S_{6}$.

Then, we can write

$$
\begin{gathered}
S_{1}+S_{5}=S_{1}+S_{4}+\frac{1}{p}\left(1-\alpha_{2}\right)<0, \quad S_{1}+S_{6}=S_{1}+S_{4}+\frac{1}{p}\left(1-\beta_{2}\right)<0 \\
S_{2}+S_{5}=S_{2}+S_{4}-\frac{1}{p}\left(1-\alpha_{2}\right)<0, \quad S_{2}+S_{6}=S_{2}+S_{6}-\left(1-\beta_{2}\right)<0 \\
S_{3}+S_{5}=S_{3}+S_{4}+\frac{1}{p}\left(1-\alpha_{2}\right)<0, \quad S_{3}+S_{6}=S_{3}+S_{4}+\frac{1}{p}\left(1-\beta_{2}\right)<0
\end{gathered}
$$

which, respectively, are equivalent to

$$
\alpha_{2} \geq \gamma_{2}+\frac{1}{q}\left(\gamma_{1}-1\right), \quad \beta_{2} \geq \gamma_{2}+\frac{1}{q}\left(\gamma_{1}-1\right)
$$

where

$$
\begin{gathered}
1-\frac{1}{p q} \leq \alpha_{1}+\frac{1}{p}\left(\alpha_{2}-\gamma_{2}\right)-\frac{\gamma_{1}}{p q}, \quad 1-\frac{1}{p q} \leq \alpha_{1}+\frac{1}{p}\left(\beta_{2}-\gamma_{2}\right)-\frac{\gamma_{1}}{p q}, \\
1-\frac{1}{p q} \leq \beta_{1}+\frac{1}{p}\left(\alpha_{2}-\gamma_{2}\right)-\frac{\gamma_{1}}{p q}, \quad 1-\frac{1}{p q} \leq \beta_{1}+\frac{1}{p}\left(\beta_{2}-\gamma_{2}\right)-\frac{\gamma_{1}}{p q} .
\end{gathered}
$$

Now, let

$$
\begin{array}{ll}
H_{1}=\alpha_{1}+\frac{1}{p}\left(\alpha_{2}-\gamma_{2}\right)-\frac{\gamma_{1}}{p q}, & H_{2}=\alpha_{1}+\frac{1}{p}\left(\beta_{2}-\gamma_{2}\right)-\frac{\gamma_{1}}{p q}, \\
H_{3}=\beta_{1}+\frac{1}{p}\left(\alpha_{2}-\gamma_{2}\right)-\frac{\gamma_{1}}{p q}, & H_{4}=\beta_{1}+\frac{1}{p}\left(\beta_{2}-\gamma_{2}\right)-\frac{\gamma_{1}}{p q} .
\end{array}
$$

Then, $1-\frac{1}{p q}<\min \left\{H_{1}, H_{2}, H_{3}, H_{4}\right\}$. Now, from (11),

$$
v_{0}\left(\int_{0}^{T} D_{T^{-}}^{\alpha_{2}} \varphi d t+\int_{0}^{T} D_{T^{-}}^{\beta_{2}} \varphi d t\right) \leq J_{1}^{1 / q}\left(J_{2}^{1 / q^{\prime}}+J_{3}^{1 / q^{\prime}}+J_{4}^{1 / q^{\prime}}\right),
$$

then

$$
v_{0} \int_{0}^{T} D_{T^{-}}^{\alpha_{2}} \varphi d t \leq J_{1}^{1 / q}\left(J_{2}^{1 / q^{\prime}}+J_{3}^{1 / q^{\prime}}+J_{4}^{1 / q^{\prime}}\right) .
$$

From Lemma 1, we obtain

$$
v_{0} \leq C T^{\alpha_{2}-1} J_{1}^{1 / q}\left(J_{2}^{1 / q^{\prime}}+J_{3}^{1 / q^{\prime}}+J_{4}^{1 / q^{\prime}}\right),
$$

and

where

$$
\begin{gathered}
v_{0} \leq C J_{1}^{1 / q} T^{\alpha_{2}-1}\left(T^{\left(\frac{\gamma 1}{q}-1\right)+\frac{1}{q^{\prime}}}+T^{\left(\frac{\gamma_{1}}{q}-\alpha_{2}\right)+\frac{1}{q^{\prime}}}+T^{\left(\frac{\gamma 1}{q}-\beta_{2}\right)+\frac{1}{q^{\prime}}}\right), \\
v_{0} \leq C J_{1}^{1 / q}\left(T^{\sigma_{1}}+T^{\sigma_{2}}+T^{\sigma_{3}}\right)
\end{gathered}
$$




$$
\begin{aligned}
& \sigma_{1}=\alpha_{2}-1+\left(\frac{\gamma_{1}}{q}-1\right)+\frac{1}{q^{\prime}}=\alpha_{2}-1+\frac{1}{q}\left(\gamma_{1}-1\right), \\
& \sigma_{2}=\alpha_{2}-1+\left(\frac{\gamma_{1}}{q}-\alpha_{2}\right)+\frac{1}{q^{\prime}}=\frac{1}{q}\left(\gamma_{1}-1\right), \\
& \sigma_{3}=\alpha_{2}-1+\left(\frac{\gamma_{1}}{q}-\beta_{2}\right)+\frac{1}{q^{\prime}}=\alpha_{2}-\beta_{2}+\frac{1}{q}\left(\gamma_{1}-1\right) .
\end{aligned}
$$

We note that $\sigma_{2}=\frac{1}{q}\left(\gamma_{1}-1\right)<0, \sigma_{1}=\alpha_{2}-1+\frac{1}{q}\left(\gamma_{1}-1\right)<0$. Using (5), we can easily prove that $\sigma_{3}<0$. Then, by sending $T \rightarrow \infty$, we arrive at the contradiction $0<v_{0} \leq 0$. A similar analysis can be performed by showing that $I_{1}$ is bounded, if

$$
1-\frac{1}{p q}<\min \left\{H_{1}^{\prime}, H_{2}^{\prime}, H_{3}^{\prime}, H_{4}^{\prime}\right\}
$$

where

$$
\begin{array}{ll}
H_{1}^{\prime}=\alpha_{2}+\frac{1}{p}\left(\alpha_{1}-\gamma_{1}\right)-\frac{\gamma_{2}}{p q}, & H_{2}^{\prime}=\alpha_{2}+\frac{1}{p}\left(\beta_{1}-\gamma_{1}\right)-\frac{\gamma_{2}}{p q}, \\
H_{3}^{\prime}=\beta_{2}+\frac{1}{p}\left(\alpha_{1}-\gamma_{1}\right)-\frac{\gamma_{2}}{p q}, & H_{4}^{\prime}=\beta_{2}+\frac{1}{p}\left(\beta_{1}-\gamma_{1}\right)-\frac{\gamma_{2}}{p q},
\end{array}
$$

which, via (10), leads to the contradiction: $0<u_{0} \leq 0$. This completes the proof of Theorem 1.

Theorem 2. Assume that

$$
\max \left\{\gamma_{1}, \gamma_{2}\right\} \leq \min \left\{\alpha_{i}, \beta_{i}\right\} \text { for } i=1,2
$$

Then problem (1) does not have a global solution.

Proof. Let us prove by contradiction. Assume that $T_{\max }=+\infty$, and consider the test function used in [7]:

$$
\varphi(t)=\left\{\begin{array}{ll}
(1-t / T)^{\lambda}, & 0 \leq t \leq T, \\
0, & t>T,
\end{array} \quad \lambda>\max \left(\gamma_{1}, \gamma_{2}\right)\right.
$$

Then, we multiply the first equation of (1) by $\varphi$ and intergrate over $(0, T)$. We have

$$
\begin{gathered}
\int_{0}^{T} u_{t} \varphi(t) d t+\int_{0}^{T}\left[D_{0^{+}}^{\alpha_{1}}\left(u(t)-u_{0}\right)+D_{0^{+}}^{\beta_{1}}\left(u(t)-u_{0}\right)\right] \varphi(t) d t=\int_{0}^{T} D_{0^{+}}^{\gamma_{1}}|v|{ }^{q} \varphi(t) d t= \\
=\int_{0}^{T}|v|^{q} D_{T-}^{\gamma_{1}} \varphi(t) d t .
\end{gathered}
$$

A simple calculation gives that, for $\lambda>\gamma_{j}$

$$
D_{T-}^{\gamma_{j}} \varphi(t)=C_{\lambda, \gamma_{j}} T^{-\lambda}(T-t)^{\lambda-\gamma_{j}},
$$

where $C_{\lambda, \gamma_{j}}=\frac{\lambda \Gamma\left(\lambda-\gamma_{j}\right)}{T\left(\lambda-2 \gamma_{j+1}\right)}, j=1,2$. Since $u_{0}>0$ and $\varphi \in C^{1}$, then we integrate by parts and have

$\int_{0}^{T}|v|{ }^{q} C_{\lambda, \gamma_{1}}(T-t)^{-\gamma_{1}} \varphi(t) d t \leq-\int_{0}^{T} u \varphi^{\prime}(t) d t+\int_{0}^{T} u(t) D_{T-}^{\alpha_{1}} \varphi(t) d t+\int_{0}^{T} u(t) D_{T-}^{\beta_{1}} \varphi(t) d t$.

Let $p^{\prime}$ be a conjugate of $p$, then 


$$
\begin{gathered}
\int_{0}^{T} u\left(-\varphi^{\prime}(t)\right) d t=\int_{0}^{T} u \varphi(t)^{1 / p} \varphi(t)^{-1 / p} \times(T-t)^{-\gamma_{2} / p}(T-t)^{\gamma_{2} / p}\left(-\varphi^{\prime}(t)\right) d t \leq \\
\leq \frac{C_{\lambda, \gamma_{2}}}{4} \int_{0}^{T}|u|^{p}(T-t)^{-\gamma_{2}} \varphi(t) d t+\left(\frac{4}{C_{\lambda, \gamma_{2}}}\right)^{p^{\prime} / p} \int_{0}^{T}(\varphi(t))^{-p^{\prime} / p}(T-t)^{\frac{\gamma_{2} p^{\prime}}{p}}\left|\varphi^{\prime}(t)\right|^{p^{\prime}} d t,
\end{gathered}
$$

and

$$
\begin{aligned}
& \int_{0}^{T} u(t) D_{T-}^{\alpha_{1}} \varphi(t) d t=\int_{0}^{T} u(t) \varphi(t)^{1 / p} \varphi(t)^{-1 / p} \times(T-t)^{-\gamma_{2} / p}(T-t)^{\gamma_{2} / p} D_{T-}^{\alpha_{1}} \varphi(t) d t \leq \\
& \leq \frac{C_{\lambda, \gamma_{2}}}{4} \int_{0}^{T}|u|^{p}(T-t)^{-\gamma_{2}} \varphi(t) d t+\left(\frac{4}{C_{\lambda, \gamma_{2}}}\right)^{p^{\prime} / p} \int_{0}^{T}(\varphi(t))^{-p^{\prime} / p}(T-t)^{\frac{\gamma_{2} p^{\prime}}{p}}\left|D_{T-}^{\alpha_{1}} \varphi(t)\right|^{p^{\prime}} d t, \\
& \int_{0}^{T} u(t) D_{T-}^{\beta_{1}} \varphi(t) d t=\int_{0}^{T} u(t) \varphi(t)^{1 / p} \varphi(t)^{-1 / p} \times(T-t)^{-\gamma_{2} / p}(T-t)^{\gamma_{2} / p} D_{T-}^{\beta_{1}} \varphi(t) d t \leq \\
& \leq \frac{C_{\lambda, \gamma_{2}}}{4} \int_{0}^{T}|u|^{p}(T-t)^{-\gamma_{2}} \varphi(t) d t+\left(\frac{4}{C_{\lambda, \gamma_{2}}}\right)^{p^{\prime} / p} \int_{0}^{T}(\varphi(t))^{-p^{\prime} / p}(T-t)^{\frac{\gamma_{2} p^{\prime}}{p}}\left|D_{T-}^{\beta_{1}} \varphi(t)\right|^{p^{\prime}} d t .
\end{aligned}
$$

Using the same steps, we find

$$
\begin{gathered}
C_{\lambda, \gamma_{1}} \int_{0}^{T}|v|^{q}(T-t)^{-\gamma_{1}} \varphi(t) d t \leq \frac{3 C_{\lambda, \gamma_{2}}}{4} \int_{0}^{T}|u|^{p}(T-t)^{-\gamma_{2}} \varphi(t) d t+ \\
+\left(\frac{4}{C_{\lambda, \gamma_{2}}}\right)^{p^{\prime} / p} \int_{0}^{T}(\varphi(t))^{-p^{\prime} / p}(T-t)^{\frac{-\gamma_{2} p^{\prime}}{p}}\left|\varphi^{\prime}(t)\right|^{p^{\prime}} d t+ \\
+\left(\frac{4}{C_{\lambda, \gamma_{2}}}\right)^{\frac{p^{\prime}}{p}} \int_{0}^{T}(\varphi(t))^{\frac{-p^{\prime}}{p}}(T-t)^{\frac{-\gamma_{2} p^{\prime}}{p}}\left(\left|D_{T-}^{\alpha_{1}} \varphi(t)\right|^{p^{\prime}}+\left|D_{T-}^{\beta_{1}} \varphi(t)\right|^{p^{\prime}}\right) d t .
\end{gathered}
$$

Let $q^{\prime}$ be a conjugate of $q$, then

$$
\begin{gathered}
C_{\lambda, \gamma_{2}} \int_{0}^{T}|u|^{p}(T-t)^{-\gamma_{2}} \varphi(t) d t \leq \frac{3 C_{\lambda, \gamma_{1}}}{4} \int_{0}^{T}|v|^{q}(T-t)^{-\gamma_{1}} \varphi(t) d t+ \\
+\left(\frac{4}{C_{\lambda, \gamma_{1}}}\right)^{q^{\prime} / q} \int_{0}^{T}(\varphi(t))^{-q^{\prime} / q}(T-t)^{\frac{-\gamma_{1} q^{\prime}}{q}}\left|\varphi^{\prime}(t)\right|^{q^{\prime}} d t+ \\
+\left(\frac{4}{C_{\lambda, \gamma_{1}}}\right)^{\frac{q^{\prime}}{q}} \int_{0}^{T}(\varphi(t))^{\frac{-q^{\prime}}{q}}(T-t)^{\frac{-\gamma_{1} q^{\prime}}{q}}\left(\left|D_{T-}^{\alpha_{2}} \varphi(t)\right|^{q^{\prime}}+\left|D_{T-}^{\beta_{2}} \varphi(t)\right|^{q^{\prime}}\right) d t .
\end{gathered}
$$

We put

$$
\begin{array}{ll}
A:=C_{\lambda, \gamma_{1}} \int_{0}^{T}|v|^{q}(T-t)^{-\gamma_{1}} \varphi(t) d t ; & C:=\int_{0}^{T}(\varphi(t))^{-p^{\prime} / p}(T-t)^{\frac{-\gamma_{2} p^{\prime}}{p}}\left|\varphi^{\prime}(t)\right|^{p^{\prime}} d t ; \\
B:=C_{\lambda, \gamma_{2}} \int_{0}^{T}|u|^{p}(T-t)^{-\gamma_{2}} \varphi(t) d t ; & D:=\int_{0}^{T}(\varphi(t))^{-q^{\prime} / q}(T-t)^{\frac{-\gamma_{1} q^{\prime}}{q}}\left|\varphi^{\prime}(t)\right|^{q^{\prime}} d t ;
\end{array}
$$




$$
\begin{aligned}
& E:=\int_{0}^{T}(\varphi(t))^{\frac{-p^{\prime}}{p}}(T-t)^{\frac{-\gamma_{2} p^{\prime}}{p}}\left(\left|D_{T-}^{\alpha_{1}} \varphi(t)\right|^{p^{\prime}}+\left|D_{T-}^{\beta_{1}} \varphi(t)\right|^{p^{\prime}}\right) d t \\
& F:=\int_{0}^{T}(\varphi(t))^{\frac{-q^{\prime}}{q}}(T-t)^{\frac{-\gamma_{1} q^{\prime}}{q}}\left(\left|D_{T-}^{\alpha_{2}} \varphi(t)\right|^{q^{\prime}}+\left|D_{T-}^{\beta_{2}} \varphi(t)\right|^{q^{\prime}}\right) d t .
\end{aligned}
$$

From (23) and (24), we have

$$
A \leq \frac{3}{4} B+\left(\frac{4}{C_{\lambda, \gamma_{2}}}\right)^{p^{\prime} / p}(C+E), \quad B \leq \frac{3}{4} A+\left(\frac{4}{C_{\lambda, \gamma_{1}}}\right)^{q^{\prime} / q}(D+F) .
$$

Therefore,

$$
\begin{aligned}
A & \leq \frac{12}{7}\left(\frac{4}{C_{\lambda, \gamma_{1}}}\right)^{q^{\prime} / q}(D+F)+\frac{16}{7}\left(\frac{4}{C_{\lambda, \gamma_{2}}}\right)^{p^{\prime} / p}(C+E), \\
B & \leq \frac{12}{7}\left(\frac{4}{C_{\lambda, \gamma_{2}}}\right)^{p^{\prime} / p}(C+E)+\frac{16}{7}\left(\frac{4}{C_{\lambda, \gamma_{1}}}\right)^{q^{\prime} / q}(D+F) .
\end{aligned}
$$

Using integration by parts in system (1) and (25), we write

$$
\begin{aligned}
& u_{0} \int_{0}^{T} D_{T-}^{\alpha_{1}} \varphi(t) d t \leq \frac{12}{7}\left(\frac{4}{C_{\lambda, \gamma_{1}}}\right)^{q^{\prime} / q}(D+F)+\frac{16}{7}\left(\frac{4}{C_{\lambda, \gamma_{2}}}\right)^{p^{\prime} / p}(C+E), \\
& v_{0} \int_{0}^{T} D_{T-}^{\alpha_{2}} \varphi(t) d t \leq \frac{12}{7}\left(\frac{4}{C_{\lambda, \gamma_{2}}}\right)^{p^{\prime} / p}(C+E)+\frac{16}{7}\left(\frac{4}{C_{\lambda, \gamma_{1}}}\right)^{q^{\prime} / q}(D+F) .
\end{aligned}
$$

Using Lemma 2, we find

$$
\begin{array}{ll}
C \leq c T^{-\gamma_{2} \frac{p^{\prime}}{p}+1-p^{\prime}} ; & D \leq c T^{-\gamma_{1} \frac{q^{\prime}}{q}+1-q^{\prime}} ; \\
E \leq c\left(T^{-\gamma_{2} \frac{p^{\prime}}{p}+1-p^{\prime} \alpha_{1}}+T^{-\gamma_{2} \frac{p^{\prime}}{p}+1-p^{\prime} \beta_{1}}\right) ; & F \leq c\left(T^{-\gamma_{1} \frac{q^{\prime}}{q}+1-q^{\prime} \alpha_{2}}+T^{-\gamma_{1} \frac{q^{\prime}}{q}+1-q^{\prime} \beta_{2}}\right)
\end{array}
$$

for some positive constant $c$. Consequently, we find

$$
\begin{aligned}
u_{0} \int_{0}^{T} D_{T-}^{\alpha_{1}} \varphi(t) d t \leq & \frac{12}{7}\left(\frac{4}{C_{\lambda, \gamma_{1}}}\right)^{q^{\prime} / q} c\left[T^{-\gamma_{1} \frac{q^{\prime}}{q}+1-q^{\prime}}+\left(T^{-\gamma_{1} \frac{q^{\prime}}{q}+1-q^{\prime} \alpha_{2}}+T^{-\gamma_{1} \frac{q^{\prime}}{q}+1-q^{\prime} \beta_{2}}\right)\right]+ \\
& +\frac{16}{7}\left(\frac{4}{C_{\lambda, \gamma_{2}}}\right)^{p^{\prime} / p} c\left[T^{-\gamma_{2} \frac{p^{\prime}}{p}+1-p^{\prime}}+\left(T^{-\gamma_{2} \frac{p^{\prime}}{p}+1-p^{\prime} \alpha_{1}}+T^{-\gamma_{2} \frac{p^{\prime}}{p}+1-p^{\prime} \beta_{1}}\right)\right] .
\end{aligned}
$$

Therefore,

$$
u_{0} \leq C_{\alpha_{1}, \lambda}^{-1} c\left[\begin{array}{c}
\frac{12}{7}\left(\frac{4}{C_{\lambda, \gamma_{1}}}\right)^{q^{\prime} / q}\left(T^{-\gamma_{1} \frac{q^{\prime}}{q}+\alpha_{1}-q^{\prime}}+\left(T^{-\gamma_{1} \frac{q^{\prime}}{q}+\alpha_{1}-q^{\prime} \alpha_{2}}+T^{-\gamma_{1} \frac{q^{\prime}}{q}+\alpha_{1}-q^{\prime} \beta_{2}}\right)\right)+ \\
+\frac{16}{7}\left(\frac{4}{C_{\lambda, \gamma_{2}}}\right)^{p^{\prime} / p}\left(T^{-\gamma_{2} \frac{p^{\prime}}{p}+\alpha_{1}-p^{\prime}}+\left(T^{-\gamma_{2} \frac{p^{\prime}}{p}+\alpha_{1}-p^{\prime} \alpha_{1}}+T^{-\gamma_{2} \frac{p^{\prime}}{p}+\alpha_{1}-p^{\prime} \beta_{1}}\right)\right)
\end{array}\right] .
$$


$v_{0} \leq C_{\alpha_{2}, \lambda}^{-1} c\left[\begin{array}{c}\frac{12}{7}\left(\frac{4}{C_{\lambda, \gamma_{2}}}\right)^{p^{\prime} / p}\left(T^{-\gamma_{2} p^{\prime} / p+\alpha_{2}-p^{\prime}}+\left(T^{\gamma_{2} p^{\prime} / p+\alpha_{2}-p^{\prime} \alpha_{1}}+T^{\gamma_{2} p^{\prime} / p+\alpha_{2}-p^{\prime} \beta_{1}}\right)\right)+ \\ +\frac{16}{7}\left(\frac{4}{C_{\lambda, \gamma_{1}}}\right)^{q^{\prime} / q}\left(T^{-\gamma_{1} q^{\prime} / q+\alpha_{2}-q^{\prime}}+\left(T^{-\gamma_{1} q^{\prime} / q+\alpha_{2}-q^{\prime} \alpha_{2}}+T^{-\gamma_{1} q^{\prime} / q+\alpha_{2}-q^{\prime} \beta_{2}}\right)\right)\end{array}\right]$.

Finally, using the fact that

$$
\begin{array}{lll}
-\gamma_{1} \frac{q^{\prime}}{q}+\alpha_{1}-q<0, & -\gamma_{1} \frac{q^{\prime}}{q}+\alpha_{1}-q^{\prime} \alpha_{2}<0, & -\gamma_{1} \frac{q^{\prime}}{q}+\alpha_{1}-q^{\prime} \beta_{2}<0, \\
-\gamma_{2} \frac{p^{\prime}}{p}+\alpha_{1}-p^{\prime}<0, & -\gamma_{2} \frac{p^{\prime}}{p}+\alpha_{1}-p^{\prime} \alpha_{1}<0, & -\gamma_{2} \frac{p^{\prime}}{p}+\alpha_{1}-p^{\prime} \beta_{1}<0, \\
-\gamma_{1} \frac{q^{\prime}}{q}+\alpha_{2}-q^{\prime}<0, & -\gamma_{1} \frac{q^{\prime}}{q}+\alpha_{2}-q^{\prime} \alpha_{2}<0, & -\gamma_{1} \frac{q^{\prime}}{q}+\alpha_{2}-q^{\prime} \beta_{2}<0
\end{array}
$$

and taking $T \rightarrow \infty$ we arrive at the contradiction: $0<u_{0} \leq 0$ or $0<v_{0} \leq 0$.

Remark 1. Theorems 1 and 2 remain valide for the new system

$$
\left\{\begin{aligned}
{ }^{c} D^{\alpha_{1}} u+{ }^{c} D^{\beta_{1}} u & =D_{0^{+}}^{\gamma_{1}}|v|^{q} \\
{ }^{c} D^{\alpha_{2}} v+{ }^{c} D^{\beta_{2}} v & =D_{0^{+}}^{\gamma_{2}}|u|^{p}, \quad t \in \mathbb{R}_{+} \\
u(0)=u_{0}, v(0) & =v_{0}
\end{aligned}\right.
$$

under the same initial conditions on the parameters.

Acknowledgements. The authors would like to thank the associate editor, and anonymous referees for their constructive corrections and valuable suggestions that improved the manuscript.

\section{References}

1. Abbas S. Existence of Solutions to Fractional Order Ordinary and Delay Differential Equations and Applications. Electronic Journal of Differential Equations, 2011, no. 9, pp. $1-11$.

2. Mahto L., Abbas S., Favini A. Analysis of Caputo Impulsive Fractional Order Differential Equations with Applications. International Journal of Differential Equations, 2013, vol. 2013, article ID: 704547. DOI: 10.1155/2013/704547

3. Mahto L., Abbas S. PC-Almost Automorphic Solution of Impulsive Fractional Differential Equations. Mediterranean Journal of Mathematics, 2015, vol. 12, no. 3, pp. 771-790. DOI: $10.1007 / \mathrm{s} 00009-014-0449-3$

4. Abbas S., Erturk V.S., Momani S. Dynamical Analysis of the Irving-Mullineux Oscillator Equation of Fractional Order. Signal Processing, 2014, no. 102, pp. 171-176. DOI: $10.1016 /$ j.sigpro.2014.03.019

5. Samko S.G., Kilbas A.A., Marichev O.I. Fractional Intergrals and Derivatives: Theory and Applications. Yverdon, New York, Gordon and Beach, 1993.

6. Mahto L., Abbas S. Existence and Uniqueness of Solution of Caputo Fractional Differential Equations. AIP Conference Proceedings, 2012, vol. 1479, article ID: 896. DOI: $10.1063 / 1.4756286$ 
7. Kilbas A.A., Sarivastava H.M., Trujillo J.J. Theory and Application of Fractinal Differential Equations, N.Y., Elsevier, 2016.

8. Mennouni A., Youkana A. Finite Time Blow-Up of Solutions for a Nonlinear System of Fractional Differential Equations. Electronic Journal of Differential Equations, 2017, vol. 2017, no. 152, pp. 1-15.

9. Caponetto R., Dongola G., Fortuna L., Petras I. Fractional Order Systems: Modeling and Control Applications. Singapore, World Scientific, 2010. DOI: 10.1142/7709

10. Kirk C.M., Olmestead W.E., Roberts C.A. A System of Nonlinear Volterra Equations with Blow-Up Solutions. Journal of Integral Equation Application, 2012, no. 24, pp. 377-393. DOI: 10.1216/JIE-2013-25-3-377

11. Stankovich B., Atanackovic T.M. On an Inequality Arising in Fractional Oscillator Theory. Fractional Calculus and Applied Analysis, 2004, no. 7, pp. 11-20.

12. Kirane M., Malik S.A. Profile of Blowing-Up Solution to Nonlinear System of Fractional Differential Equations. Nonlinear Analysis: Theory, Methods and Applications, 2010, vol. 73, no. 12, pp. 3723-3736. DOI: 10.1016/j.na.2010.06.088

13. Qun D., Huilai L. To Study Blowing-Up of a Non Linear System of Fractional Differential Equations. SCIENTIA SINICA Mathematica, 2012, vol. 42, no. 12, pp. 1205-1212. DOI: $10.1360 / 012011-800$

14. Kadem A., Kirane M., Kirk C.M., Olmstead W.E. Blowing-up Solutions to System of Fractional Differential and Integral Equations with Exponential Nonlinearities. IMA Journal of Applied Mathematics, 2014, vol. 79, no. 6, pp. 1077-1088. DOI: 10.1093/imamat/hxt005

Received July 27, 2019

УДК 517.9

DOI: $10.14529 / \mathrm{mmp} 200104$

\title{
РАЗРЕШЕНИЯ ДЛЯ НЕЛИНЕЙНОЙ ФРАКЦИОННОЙ ДИФФЕРЕНЦИАЛЬНОЙ СИСТЕМЫ КАПУТО
}

\author{
M. Терчи ${ }^{1}$, Х. Хассуна
}

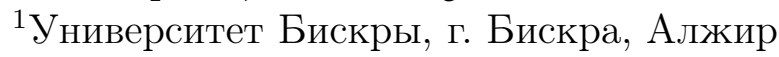

В статье устанавливается разрушение за конечное время решений нелинейных дифференциальных систем, основанных на дифференциальном уравнении Капуто. Найдены достаточные условия на параметры с положительными данными. Более того, для этой цели, при некоторых предположениях, доказано несуществование глобальных решений для рассматриваемого класса нелинейных дробно-дифференциальных систем Капуто при заданном начальном условии. Чтобы доказать основной результат, применяется метод тестовой функции, интеграл Римана - Лиувилля, оператор производной Капуто и некоторые общие инструменты анализа. Наш результат является новым и обобщает существующий.

Ключевые слова: дифференииальные уравнения; интеграл Римана - Лиувилля; дифберенциальный оператор Kaпуто.

Мессауда Терчи, лаборатория прикладной математики, Университет Бискры (г. Бискра, Алжир), terchi1.messaouda@gmail.com.

Худа Хассуна, кафедра математики, Университет Бискры (г. Бискра, Алжир), houda.hassouna@yahoo.fr.

Поступила в редакиию 27 июля 2019 г. 